\title{
Transcatheter Arterial Embolization for Late Postpancreatectomy Hemorrhage of Unusual Origin (Dorsal Pancreatic Artery): A Report of
} Three Cases

\author{
Yook Kim ${ }^{1}$, Jisun Lee ${ }^{1,{ }^{*}}$, Kyung Sik Yi ${ }^{1}$, Soo Hyun Lee ${ }^{1}$, Bum Sang Cho ${ }^{1,2}$ and Kil Sun Park ${ }^{1,2}$ \\ ${ }^{1}$ Department of Radiology, Chungbuk National University Hospital, Cheongju, Republic of Korea \\ ${ }^{2}$ Department of Radiology, College of Medicine, Chungbuk National University, Cheongju, Republic of Korea \\ "Corresponding author: Department of Radiology, Chungbuk National University Hospital, 776, 1Sunhwan-ro, Seowon-gu, Cheongju, \\ Chungcheongbuk-do, 28644, Republic of Korea. Tel: +82-432696477, Fax: +82-432696479, Email: dr.jisunlee@gmail.com \\ Received 2018 July 22; Revised 2018 December 27; Accepted 2018 December 29.
}

\begin{abstract}
Postpancreatectomy hemorrhage (PPH) is a rare but serious complication that can lead to death. Although the best treatment option for PPH remains controversial, transcatheter arterial embolization(TAE) has recently been introduced as a treatment with safety and efficacy, showing low morbidity and mortality, especially in late PPH (> $24 \mathrm{~h}$ after surgery). The most common location for PPH is the gastroduodenal artery stump; however, hemorrhage may occur from unusual sources, such as the dorsal pancreatic artery (DPA), which is often difficult to detect on angiography. Herein, the authors report three cases of delayed PPH from the DPA, occurring after a Frey procedure in one case and after pylorus-preserving pancreaticoduodenectomy in the other two. All patients were successfully treated using TAE. Although the involved artery was not identified on the first angiography in two patients, rebleeding was successfully managed by repeated endovascular procedures. Therefore, a careful and repetitive approach, based on awareness of the various causes of PPH, could increase the success rate of TAE.
\end{abstract}

Keywords: Pancreatectomy, Hemorrhage, Embolization, Therapeutic, Arteries, Anatomic Variation, Dorsal Pancreatic Artery (DPA)

\section{Introduction}

Postpancreatectomy hemorrhage $(\mathrm{PPH})$ is a rare $(4 \%$ - 14\%) but potentially fatal complication and the PPHassociated mortality has been reported to be between $14.3 \%$ and $30.7 \%$ (1-5). Immediate detection of bleeding and proper treatment, therefore, are crucial in reducing mortality. PPH is divided into two phases according to the elapsed time after surgery. Early hemorrhage ( $\leq 24 \mathrm{~h}$ after surgery) usually occurs due to technical failure and requires immediate repeated laparotomy. Late hemorrhage ( $>24 \mathrm{~h}$ after surgery) can be accompanied by pancreatic leak or pseudoaneurysm around the surgical site due to erosion of the vessels (6). Although the best treatment option for PPH remains controversial, transcatheter arterial embolization (TAE) has recently been introduced as a treatment with safety and efficacy, showing low morbidity and mortality, especially in late $\mathrm{PPH}(2,3,7-12)$. The most common location for delayed PPH is the gastroduodenal artery (GDA) stump, followed by the splenic, hep- atic, and gastric arteries. In addition to these, unusual sources of hemorrhage, including the dorsal pancreatic artery (DPA), jejunal, phrenic, or adrenal arteries, may also be observed $(2,3,8,9)$. Therefore, awareness of the potential for bleeding from rare sources is important. Among these, TAE for the DPA is technically more challenging-and sometimes even impossible-because it is difficult to predict the origin and localize the artery on angiography due to the unfavorable vascular anatomy. There have been reports on cases where TAE failed, requiring repetitive laparotomy to control bleeding $(13,14)$. Herein, we report three cases of delayed PPH from the DPA, occurring after a Frey procedure-local resection of the head of pancreas combined with longitudinal pancreaticojejunostomy to treat the chronic pancreatitis-in one case and after pyloruspreserving pancreaticoduodenectomy (PPPD) in the other two, which were successfully treated using TAE in all cases. 


\section{Case Presentation}

\subsection{Case 1}

A 53-year-old male underwent a Frey procedure for chronic calcific pancreatitis. However, on postoperative day 3 , bloody drainage from the surgical wound was observed. On postoperative day 5 , he experienced melena, and the hemoglobin level declined from $12.1 \mathrm{~g} / \mathrm{dL}$ to 10.3 g/dL. However, computed tomography (CT) scan revealed no evidence of bleeding, except for peripancreatic fluid collection. On postoperative day 7 , the amount of fluid from the drainage tube was markedly increased from 70 $\mathrm{mL}$ to $620 \mathrm{~mL}$, and exhibited a fresh bloody color. In addition, his hemoglobin level declined from $9.9 \mathrm{~g} / \mathrm{dL}$ to $6.1 \mathrm{~g} / \mathrm{dL}$ during a $2 \mathrm{~h}$ period, which necessitated emergent packed red blood cell transfusion. Angiography was performed as a primary diagnostic and therapeutic procedure. However, because no involved artery was identified on celiac and superior mesenteric artery (SMA) angiographies, immediate treatment could not be performed. The following day, fresh blood $(715 \mathrm{~mL}$ ) continued to drain from the drainage tube; therefore, repeated dynamic CT scan was performed. A $9 \times 7 \mathrm{~mm}$ pseudoaneurysm was revealed in the pancreatic bed-probably from a branch of the SMAon the arterial phase of a follow-up CT scan (Figure 1A). Subsequently, a second endovascular procedure was attempted, and SMA angiography using automated injector (30 mL of iodinated contrast material at a rate of 6 $\mathrm{mL} / \mathrm{s}$ ), which was more than the amount of contrast material used in the initial angiography $(15 \mathrm{~mL}$ at a rate of $3 \mathrm{~mL} / \mathrm{s}$ ), demonstrated the pseudoaneurysm arising from a branch of the DPA (transverse pancreatic artery) (Figure $1 \mathrm{~B})$. Selective angiography of the DPA using a microcatheter (2.2 Fr; Progreat, Terumo Interventional Systems, Somerset, NJ, USA) revealed the pseudoaneurysm corresponding to the CT finding (Figure 1C). Embolization was attempted with six fibered platinum coils ( $\operatorname{six} 3 / 2 \mathrm{~mm}$ coils; Tornado Embolization Coil; Cook Medical Inc, Bloomington, IN, USA), which were deployed distal and proximal to the pseudoaneurysm. A post-embolization angiography demonstrated successful hemostasis with exclusion of the pseudoaneurysm (Figure1D). Pancreatic enzyme levels and hemoglobin recovered to normal ranges after the procedure and no more complications developed. A CT scan performed two weeks after the procedure demonstrated complete occlusion of the pseudoaneurysm. The patient underwent subsequent CT scans during the 36-month followup period, which demonstrated no recurrence of the pseudoaneurysm.

\subsection{Case 2}

An 80-year-old female underwent pylorus preserving pancreaticoduodenectomy (PPPD) for a distal common bile duct cancer without immediate complications. On postoperative day 5 , she experienced melena, and her hemoglobin level declined from $11.0 \mathrm{~g} / \mathrm{dL}$ to $6.7 \mathrm{~g} / \mathrm{dL}$. Postoperative conventional abdominal CT scan demonstrated collection of contrast material in the lumen of pancreaticojejunostomy site adjacent to the splenic artery suggesting an extravasation (Figure $2 \mathrm{~A}$ ). She was referred to the department of interventional radiology for definitive treatment. The celiac axis was selected for angiography to confirm active extravasation of contrast material in the proximal portion of the celiac trunk. Celiac angiography showed a leakage of amorphous contrast agent around the splenic artery suggesting an extravasation. However, a culprit artery was not clearly identified (Figure 2B). Multiple selective angiographies using a microcatheter (2.2 Fr; Progreat, Terumo) were performed on each branch of the celiac trunk to find the bleeding site, which were the common hepatic artery and from distal splenic artery to proximal splenic artery. Finally, stagnation of contrast media suggestive of active bleeding was found on proximal splenic angiography (Figure $2 \mathrm{C}$ ). Given the CT finding in which the splenic artery was intact without vessel irregularity or caliber change, it was predicted that a branch, such as the DPA arising from the splenic artery, was the most likely bleeding focus rather than the splenic artery itself. Therefore, endovascular trapping of the proximal splenic artery was performed using 16 platinum coils (six $8 / 4 \mathrm{~mm}$ coils, five $6 / 2 \mathrm{~mm}$, and five $4 / 2 \mathrm{~mm}$; Tornado embolization coil, Cook Medical Inc.). A post-embolization angiography demonstrated successful hemostasis with no residual contrast staining (Figure 2D). No more complications developed and the patient was discharged two weeks later. The patient underwent a follow-up CT scan at 48 months and was doing well without any abnormal findings.

\subsection{Case 3}

A 69-year-old male underwent PPPD for an ampulla of Vater cancer. On postoperative day 21, $300 \mathrm{~mL}$ of blood was present in the drainage bag and a drop $>3.0 \mathrm{~g} / \mathrm{dL}$ in hemoglobin level necessitated a 5-unit blood transfusion. CT scan revealed a large hematoma in the jejunal loop, without evidence of active extravasation or pseudoaneurysm. Celiac angiography revealed focal bulging at the GDA stump of the hepatic artery, which was believed to be a pseudoaneurysm and the bleeding focus (Figure $3 \mathrm{~A}$ ). 

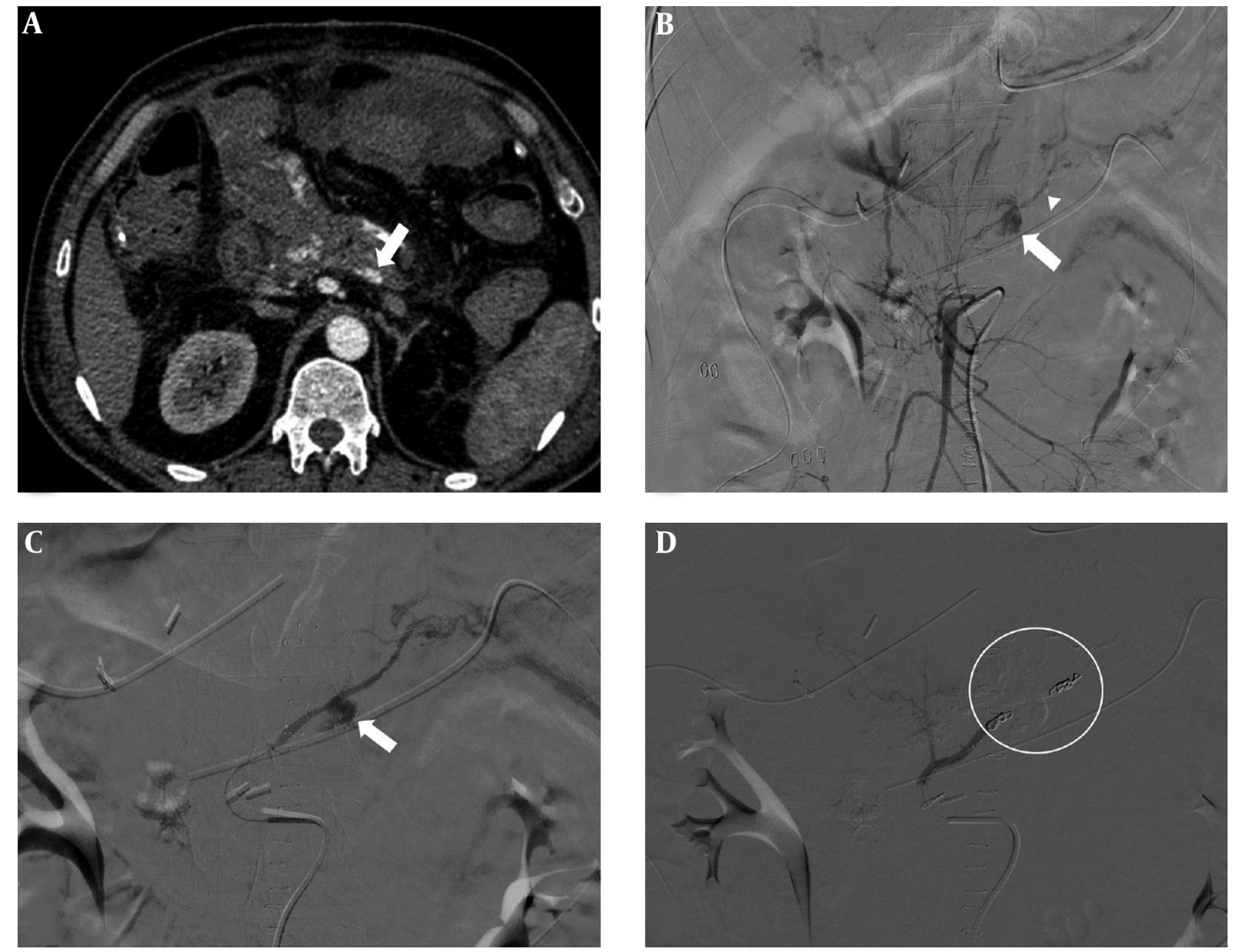

Figure 1. A 53-year-old male who experienced post-pancreatectomy hemorrhage after a Frey procedure due to chronic pancreatitis. A, On postoperative day 8, computed tomography (CT) revealed a $9 \times 7 \mathrm{~mm}$ pseudoaneurysm (arrow) in the pancreatic bed, probably from a branch of the superior mesenteric artery (SMA), on arterial phase. B, SMA angiography revealed the pseudoaneurysm (arrow) arising from a branch of the dorsal pancreatic artery (DPA) (transverse pancreatic artery, arrowhead). C, A selective angiography of the DPA using a microcatheter revealed the pseudoaneurysm (arrow) corresponding to the CT finding. D, Embolization was attempted with six fibered platinum coils, which were deployed distal and proximal to the pseudoaneurysm. Post-embolization angiography confirmed successful hemostasis with exclusion of the pseudoaneurysm (circle).

It was decided to deploy a covered stent graft at the hepatic artery to treat the pseudoaneurysm of the GDA stump (Figure 3B). After placement of the stent, follow-up angiography demonstrated that no residual focal bulging lesion remained along the hepatic artery (Figure 3C). Three days after the first TAE, the patient experienced hematemesis and was hemodynamically unstable. Although a second endovascular procedure was attempted, no evidence of active bleeding or pseudoaneurysm on celiac angiography was observed. However, during the catheterization of the splenic artery, the blood flow to the splenic artery was reduced due to incidental vasospasm, and the pseudoaneurysm at the DPA arising from proximal splenic artery, which had not been just previously evident, appeared (Fig- ure 3D). The DPA was superselectively catheterized using a microcatheter (2.2 Fr; Progreat, Terumo). Two fibered platinum coils (two 3/2 mm coils; Tornado embolization coil, Cook Medical Inc) were deployed distal to the pseudoaneurysm, and further embolization was performed with a 1:2 mixture of N-butyl cyanoacrylate (NBCA) and iodized oil at the proximal portion of pseudoaneurysm (Figure 3E). A post-embolization angiogram revealed successful hemostasis with exclusion of the pseudoaneurysm (Figure 3F). The patient experienced no further episodes of bleeding, but gradually developed sepsis and died of multiorgan failure 25 days after PPPD. 

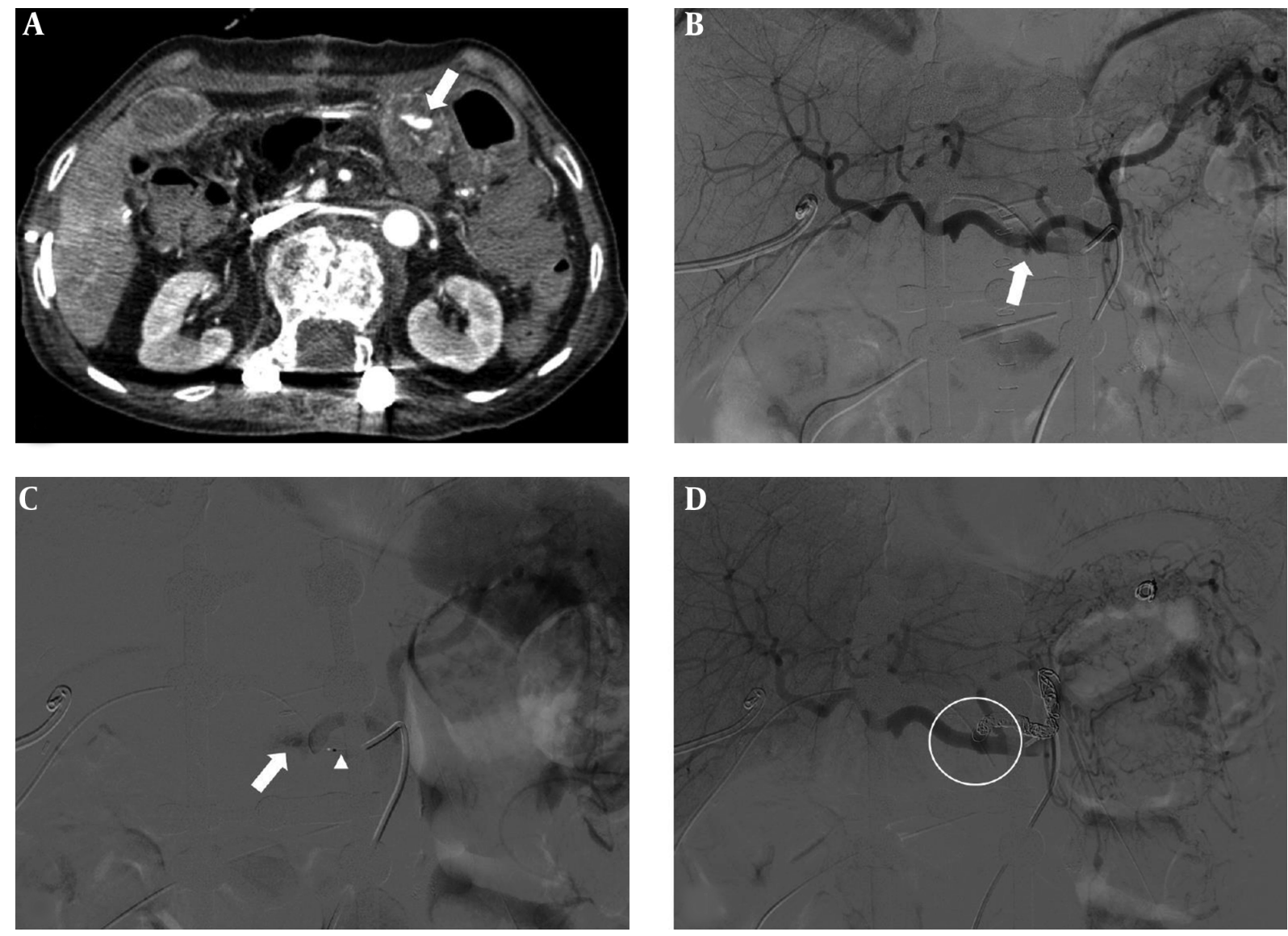

Figure 2. An 80-year-old female who experienced postpancreatectomy hemorrhage after pylorus-preserving pancreaticoduodenectomy due to a distal common bile duct cancer. A, On postoperative day 5, computed tomography(CT) scan demonstrated collection of contrast material in the lumen of pancreaticojejunostomy site (arrow) adjacent to the splenic artery suggesting an extravasation. B, The celiac axis was selected for angiography to confirm active extravasation of contrast material (arrow) in the proximal portion of the celiac trunk; celiac angiography showed a leakage of amorphous contrast agent around the splenic artery suggesting an extravasation, however a culprit artery was not clearly identified. C, Multiple selective angiographies using a microcatheter were performed on each branch of the celiac trunk to find the bleeding site, which were the common hepatic artery and from distal splenic artery to proximal splenic artery. A coaxial microcatheter system seated in the origin of splenic artery (arrowhead). Finally, stagnation of contrast media suggestive of active bleeding was found on proximal splenic angiography (arrow). D, Endovascular trapping of the proximal splenic artery was performed using 16 platinum coils. Post-embolization angiography demonstrated no residual contrast staining (circle).

\section{Discussion}

PPH has been classified as early hemorrhage $(\leq 24 \mathrm{~h})$ and late hemorrhage ( $>24 \mathrm{~h}$ ) over time by the international study group of pancreatic surgery (ISGPS) (6). Early PPH usually occurs due to technical failure and requires immediate repeated laparotomy. Late PPH is accompanied by vessel erosion in cases involving pancreatic leak or pseudoaneurysm formation in the postoperative interval (611). In our cases, late $\mathrm{PPH}$ with anastomotic leak from the drainage tube was identified in all patients. Local complications, such as abscess caused by anastomotic leak, potentially contributed to the development of delayed PPH and was confirmed by CT scan in two patients (cases 1 and 2), and a large hematoma in the jejunal loop was revealed on
CT imaging in one patient (case 3) (Table 1). PPHs are classified into three grades depending on the time of onset and severity of the bleeding. Grade A is defined as PPH that occurs early ( $\leq 24 \mathrm{~h}$ ) and has no significant clinical impact. And grades B and C are defined as PPHs that occur late (> $24 \mathrm{~h}$ ) and require diagnostic tests and appropriate management. A decrease in serum hemoglobin $<3 \mathrm{~g} / \mathrm{dL}$ is considered a grade $\mathrm{B}$ while a decrease $>3 \mathrm{~g} / \mathrm{dL}$ is considered a grade C. (6). In our cases, delayed PPH occurred, on average, on postoperative day 17 (range, 15 - 21 days), and serum hemoglobin levels were reduced by more than $3 \mathrm{~g} / \mathrm{dL}$ in all patients, which were classified as grade C PPHs and were treated successfully using TAE (Table 1 ).

The most common site for delayed PPH is known as 

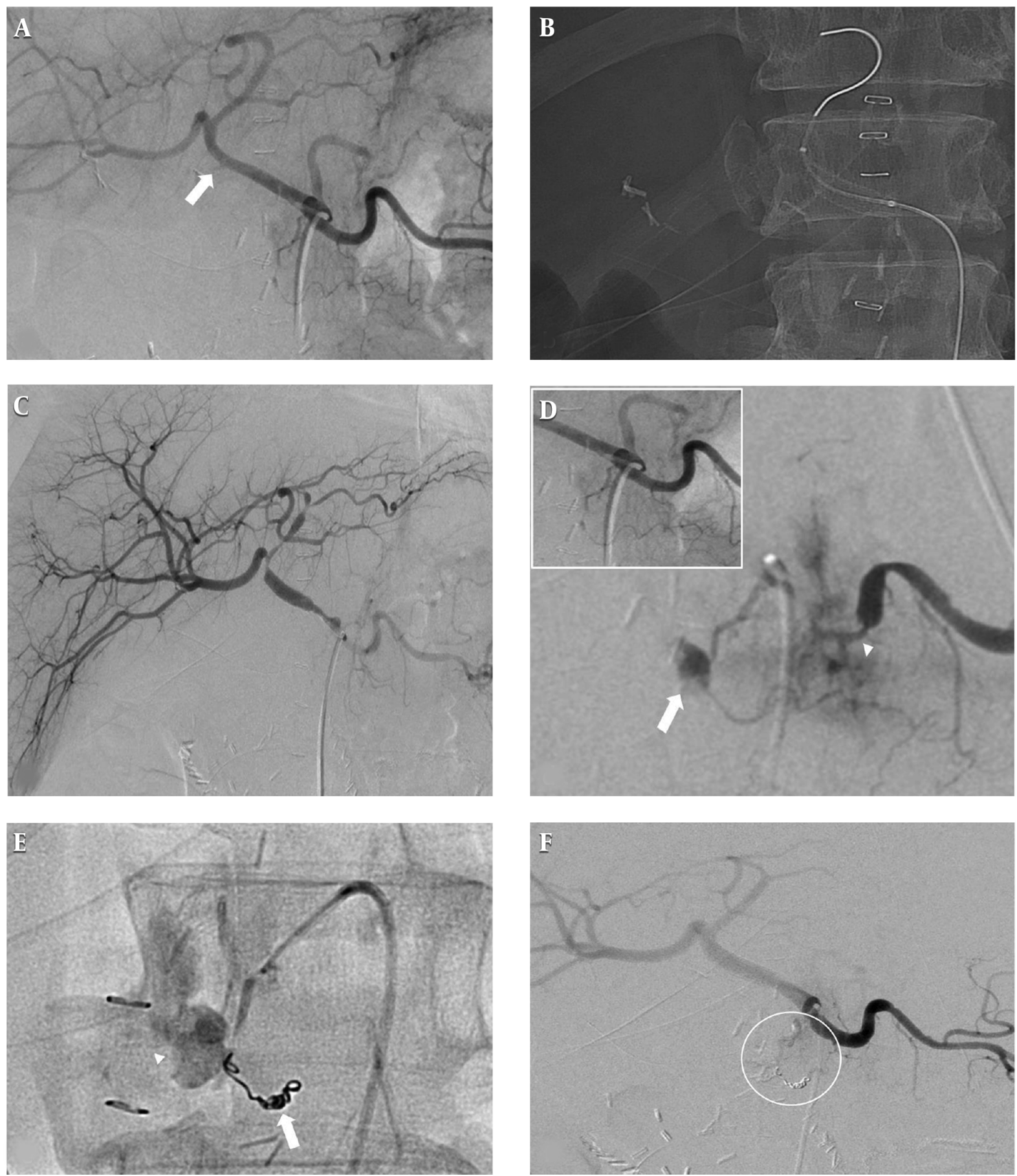

Figure 3. A 69-year-old male who experienced postpancreatectomy hemorrhage after pylorus-preserving pancreaticoduodenectomy due to ampulla of Vater cancer. A, On postoperative day 21, celiac angiography revealed focal bulging (arrow) at the gastroduodenal artery (GDA) stump of the hepatic artery, suggesting pseudoaneurysm as the bleeding focus. B, It was decided to deploy the covered stent graft at the hepatic artery to treat the pseudoaneurysm of the GDA stump. C, After placement of the stent, follow-up angiography revealed that no residual focal bulging lesion remained along the hepatic artery. D, Three days after the first transcatheter arterial embolization (TAE), a second endovascular procedure was attempted because rebleeding was suspected. During the catheterization of the splenic artery, the blood flow to the splenic artery was reduced due to incidental vasospasm (arrowhead), and the pseudoaneurysm (arrow) at the dorsal pancreatic artery (DPA) arising from proximal splenic artery, which had not been evident three days previously (figure in white-border box on top left), appeared. E, The DPA was superselectively catheterized using a microcatheter. Two fibered platinum coils (arrow) were deployed distal to the pseudoaneurysm and further embolization was performed with a 1:2 mixture of N-butyl cyanoacrylate and iodized oil (arrowhead) at the proximal portion of pseudoaneurysm. F, Post-embolization angiogram confirmed successful hemostasis with exclusion of the pseudoaneurysm (circle). 


\begin{tabular}{|c|c|c|c|c|c|c|c|c|c|c|c|c|}
\hline \multirow[t]{2}{*}{ Case } & \multirow[t]{2}{*}{ Age, $y$} & \multirow[t]{2}{*}{ Sex } & \multirow[t]{2}{*}{ Operation } & \multirow[t]{2}{*}{ POD } & \multirow[t]{2}{*}{ Presentation } & \multirow{2}{*}{$\begin{array}{c}\text { PPH } \\
\text { grade }^{a}\end{array}$} & \multirow{2}{*}{$\begin{array}{c}\text { Pancreatic } \\
\text { leak }\end{array}$} & \multirow{2}{*}{$\begin{array}{l}\text { Origin of } \\
\text { DPA }\end{array}$} & \multirow{2}{*}{$\begin{array}{c}\text { Embolic } \\
\text { material }\end{array}$} & \multicolumn{2}{|c|}{ Outcome } & \multirow{2}{*}{$\begin{array}{c}\text { Follow-up, } \\
\text { d }\end{array}$} \\
\hline & & & & & & & & & & Hemostasis & Death & \\
\hline $\mathbf{1}$ & 53 & Male & $\begin{array}{c}\text { Frey } \\
\text { procedure }\end{array}$ & 15 & Melena & $\mathrm{C}$ & Yes & SMA & Coil & Yes & No & 1080 \\
\hline 2 & 80 & Fema & PPPD & 15 & Melena & $\mathrm{C}$ & Yes & $\begin{array}{l}\text { Splenic } \\
\text { artery }\end{array}$ & Coil & Yes & No & 1440 \\
\hline 3 & 69 & Male & PPPD & 21 & Hematemesis & $\mathrm{C}$ & Yes & $\begin{array}{c}\text { Splenic } \\
\text { artery }\end{array}$ & $\begin{array}{l}\text { Coil, } \\
\text { NBCA }\end{array}$ & Yes & Yes & 25 \\
\hline
\end{tabular}

Abbreviations: d, days; DPA, dorsal pancreatic artery; NBCA, N-butyl cyanoacrylate; POD, postoperative day; PPH, postpancreatectomy hemorrhage; PPPD, pyloruspreserving pancreaticoduodenectomy; SMA, superior mesenteric artery; $y$, years

${ }^{\text {a }}$ Grade A is defined as PPH that occurs early $(\leq 24 \mathrm{~h}$ ) and has no significant clinical impact. And grades B and C are defined as PPHs that occur late (> $24 \mathrm{~h})$ and require diagnostic tests and appropriate management. A decrease in serum hemoglobin $<3 \mathrm{~g} / \mathrm{dL}$ is considered a grade $\mathrm{B}$ while a decrease $>3 \mathrm{~g} / \mathrm{dL}$ is considered a grade $\mathrm{C}$.

an eroded GDA stump by pancreatic juice (15). Less commonly, life-threatening hemorrhage may also result from injury to other arteries. Increased awareness of bleeding from unusual arteries, such as the DPA or transverse pancreatic artery from the DPA, would decrease the chances of technical failure or rebleeding rate of any radiological procedure, which necessitate re-operation. The body of the pancreas is supplied via the transverse pancreatic artery, which is the destination of the DPA. The most common origin of the DPA is the splenic artery (60\%), followed by the common hepatic artery (15\%), SMA (10\%), celiac trunk (10\%) and, rarely, others. The usual origin of the DPA is within $2 \mathrm{~cm}$ of the celiac terminus, courses down and right if it is from splenic, down and left if it is from the common hepatic artery, and up if from the SMA (16). A review of the English-language literature identified only five case reports of DPA hemorrhage after pancreatic surgery $(9,12-14)$. Among them, the origins of the DPA were four splenic arteries and one common hepatic artery. Endovascular embolization was attempted as a first treatment in all cases, and technical and clinical successes were reported in four patients. Hemostasis was not achieved by embolization in one patient; therefore, a percutaneous fluoroscopy-guided direct NBCA procedure was performed, and the pseudoaneurysm was successfully treated (13). In our cases, the origins of the DPA were one SMA and two splenic arteries, and all patients were successfully treated using TAE. To the best of our knowledge, late PPH from DPA arising from the SMA, as described in case 1 , has not been reported. Our cases support the need to confirm the possibility of hemorrhage from the DPA originating from various arteries when a definite causative artery is not apparent in angiography in patients with suspected PPH.

Multi-detector CT (MDCT) is usually used as the initial diagnostic modality for detection of bleeding focus after surgery. It may help the interventional radiologist plan for the procedure and, is thus, likely to decrease the number of angiograms required to localize the bleeding site and radiation dose received by the patient. A recent study reported that MDCT found active bleeding more frequently than angiography (9). This represents complementary roles of multiphase MDCT and angiography in postoperative management of patients undergoing pancreatectomy. The utility of MDCT in patients with unusual sites of bleeding has not been extensively studied; nevertheless, MDCT in our cases facilitated the approximate localization of the bleeding site from adjacent major vessels. In case 1, initial MDCT after surgery was negative for hemorrhage and the first angiography failed to demonstrate any area of extravasation of contrast material or pseudoaneurysm. Repeated MDCT revealed the origin of the pseudoaneurysms and the suspected branch of the hemorrhage site before angiography, which helped to find the cause of a very rare PPH, a branch of the DPA originating from the SMA. In case 2, the extravasation of contrast material in the pancreaticojejunostomy site near the splenic artery was confirmed by MDCT. Because the splenic artery itself was intact on CT imaging, it could be presumed that the branch originating from the splenic artery was damaged. Thus, MDCT would be the most useful diagnostic modality in patients with PPH and, even if there is no abnormality in the first postoperative MDCT, there should be no hesitation in performing repeat $\mathrm{CT}$ examination in cases of clinically suspected rebleeding.

Late PPH has been reported to occur into the gastrointestinal tract or more commonly, the abdominal cavity (7). Endoscopy, which is the standard first-line investigative tool for gastrointestinal bleeding, may fail because of difficulties in localizing the bleeding site in the abdominal cavity and lead to delay in TAE. Therefore, endoscopy has a very limited role in the initial management of delayed 
PPH. In the past, the first-choice treatment for PPH has been considered surgery, and even early PPH due to the technical failure of original surgery still requires immediate repeated laparotomy $(2,3,7,8,14,16)$. During re-operation, access to the bleeding vessel is limited due to postoperative inflammation, whereas endovascular treatment could be more effective in the management of PPH because rapid control of bleeding is possible. Recently, GDA stump was successfully excluded by endovascular treatments including selective embolization of the stump with coils, embolization of hepatic artery, and placement of a covered stent in the hepatic artery. Furthermore, it has been reported that endovascular treatment results in effective hemostasis with a lower morbidity and mortality than surgical care $(7,10,11)$. Okada et al. reported that selective TAE of the dorsal pancreatic artery caused only localized ischemic necrosis without clinically relevant pancreatitis on a histological change in a Swine model (17). Therefore, percutaneous endovascular treatments are considered to be the first-line management for ISGPS grade B and C PPHs.

However, TAE also has some limitations. If the origin of bleeding is a vein, or is diffuse or intermittent, the involved vessel may not be detected on angiography, which was the scenario in the first attempt in case 1 . A recent study conducted by Pottier et al. which evaluated endovascular management in patients with late $\mathrm{PPH}$, reported that the rate of rebleeding after a first endovascular treatment was as high as $43 \%$ and the only predictor related to rebleeding was abstinence (no evidence of an involved artery on angiography) or technical failure in the initial session of the radiological procedure. However, almost three-quarters of the patients were successfully treated with only endovascular treatment, and $17 \%$ of them required secondary or third procedures (7). Interestingly, cases 1 and 3 in the present study demonstrated that rebleeding occurred after the first angiography, which identified the absence of an involved artery. However, the rebleeding was successfully managed in the second endovascular procedure. In case 1 , the bleeding focus was identified after increasing the volume of contrast medium on the second angiography. Although the initial angiography did not show an obvious culprit artery in case 2, the SMA, hepatic artery, and distal portion of the splenic artery could be excluded by multiple angiographies. Finally, the DPA from the proximal portion of the splenic artery was the suspected bleeding focus, which was successfully treated using TAE. In case 3 , the first session and initial angiography of the second endovascular procedure revealed no evidence of active bleeding or pseudoaneurysm on celiac angiography. For- tunately, the pseudoaneurysm at the DPA arising from the proximal splenic artery was revealed by iatrogenic spasm, which was caused by catheterization of the splenic artery. Therefore, we recommend that the following points be considered when TAE is performed in patients with late PPH. First, when performing angiography, use as much contrast medium as possible, and try to perform selective angiography, such as splenic artery angiography, whenever possible. Second, clinicians should always be mindful of the possibility of an unusual bleeding focus of late PPH, such as DPA, and, even if a common bleeding site on the first attempt on angiography is not found, additional efforts must be made to find these other causes. Finally, if the initial procedure fails in patients with late $\mathrm{PPH}$ and the patients are hemodynamically stable, catheterization should be maintained in the femoral artery for the next 2 - 3 days. If there are signs of rebleeding, repeated endovascular procedures may be attempted before considering immediate re-operation.

In conclusion, therapeutic angiography is emerging as an effective and safe treatment strategy for PPH. The cause of bleeding after PPH is not only the well-known GDA, but also unusual origins, including the PDA, as presented in our cases, which may not be obvious in the initial angiography. Therefore, it is expected that a careful and repetitive approach, based on awareness of the various possible causes of PPH, could increase the success rate of TAE.

\section{Footnotes}

Authors' Contributions: Study concept and design: Yook Kim and Jisun Lee; analysis and interpretation of data: Yook Kim; drafting of the manuscript: Yook Kim; critical revision of the manuscript for important intellectual content: Bum Sang Cho, Kyung Sik Yi, Soo Hyun Lee, and Kil Sun Park; study supervision: Jisun Lee

Conflict of Interests: None of the authors have any potential conflicts to disclose (financial, professional, or personal) that are relevant to the manuscript.

Ethical Considerations: This retrospective study was approved by the institutional review board at our institution, and the need for informed consent was waived. The study protocol conforms to the ethical guidelines of the 1975 Declaration of Helsinki as reflected in a priori approval by the institutional human research committee.

Financial Disclosure: There was no financial disclosure for the research. 
Funding/Support: There was no funding support for the research.

\section{References}

1. Gouma DJ. Management and outcomes of haemorrhage after pancreatogastrostomy versus pancreatojejunostomy (Br J Surg 2011: 98: 1599-1607). BrJ Surg. 2011;98(11):1607-8. doi: 10.1002/bjs.7624. [PubMed: 21964685].

2. Limongelli P, Khorsandi SE, Pai M, Jackson JE, Tait P, Tierris J, et al. Management of delayed postoperative hemorrhage after pancreaticoduodenectomy: A meta-analysis. Arch Surg. 2008;143(10):1001-7. discussion 1007. doi: 10.1001/archsurg.143.10.1001. [PubMed: 18936380].

3. Yekebas EF, Wolfram L, Cataldegirmen G, Habermann CR, Bogoevski D, Koenig AM, et al. Postpancreatectomy hemorrhage: diagnosis and treatment: An analysis in 1669 consecutive pancreatic resections. Ann Surg. 2007;246(2):269-80. doi: 10.1097/01.sla.0000262953.77735.db. [PubMed: 17667506]. [PubMed Central: PMC1933568].

4. Sato N, Yamaguchi K, Shimizu S, Morisaki T, Yokohata K, Chijiiwa $\mathrm{K}$, et al. Coil embolization of bleeding visceral pseudoaneurysms following pancreatectomy: The importance of early angiography. Arch Surg. 1998;133(10):1099-102. doi: 10.1001/archsurg.133.10.1099. [PubMed: 9790208].

5. Rumstadt B, Schwab M, Korth P, Samman M, Trede M. Hemorrhage after pancreatoduodenectomy. Ann Surg. 1998;227(2):236-41. doi: 10.1097/00000658-199802000-00013. [PubMed: 9488522]. [PubMed Central: PMC1191241].

6. Wente MN, Veit JA, Bassi C, Dervenis C, Fingerhut A, Gouma DJ, et al. Postpancreatectomy hemorrhage (PPH): An International Study Group of Pancreatic Surgery (ISGPS) definition. Surgery. 2007;142(1):20-5. doi: 10.1016/j.surg.2007.02.001. [PubMed: 17629996].

7. Pottier E, Ronot M, Gaujoux S, Cesaretti M, Barbier L, Sauvanet A, et al. Endovascular management of delayed post-pancreatectomy haemorrhage. Eur Radiol. 2016;26(10):3456-65. doi: 10.1007/s00330-016-4213-x. [PubMed: 26815369].

8. Gaudon C, Soussan J, Louis G, Moutardier V, Gregoire E, Vidal V. Late postpancreatectomy hemorrhage: Predictive factors of morbidity and mortality after percutaneous endovascular treatment. $D i$ agn Interv Imaging. 2016;97(11):1071-7. doi: 10.1016/j.diii.2016.08.003. [PubMed: 27592120].
9. Stampfl U, Hackert T, Sommer CM, Klauss M, Bellemann N, Siebert S, et al. Superselective embolization for the management of postpancreatectomy hemorrhage: A single-center experience in 25 patients.J Vasc Interv Radiol. 2012;23(4):504-10. doi:10.1016/j.jvir.2011.12.013. [PubMed: 22365592].

10. Hur S, Yoon CJ, Kang SG, Dixon R, Han HS, Yoon YS, et al. Transcatheter arterial embolization of gastroduodenal artery stump pseudoaneurysms after pancreaticoduodenectomy: Safety and efficacy of two embolization techniques. J Vasc Interv Radiol. 2011;22(3):294-301. doi: 10.1016/j.jvir.2010.11.020. [PubMed: 21353982].

11. Gwon DI, Ko GY, Sung KB, Shin JH, Kim JH, Yoon HK. Endovascular management of extrahepatic artery hemorrhage after pancreatobiliary surgery: Clinical features and outcomes of transcatheter arterial embolization and stent-graft placement. AJR Am J Roentgenol. 2011;196(5):W627-34. doi: 10.2214/AJR.10.5148. [PubMed: 21512055].

12. Khorsandi SE, Limongelli P, Jackson JE, Tait P, Williamson RC, Habib NA, et al. Management of delayed arterial hemorrhage after pancreaticoduodenectomy. A case series. JOP. 2008;9(2):172-8. [PubMed: 18326925].

13. Kim RH, Yoo RE, Kim HC. Percutaneous N-butyl cyanoacrylate embolization of a pancreatic pseudoaneurysm after failed attempts of transcatheter embolization. J Korean Soc Radiol. 2014;71(4):173. doi: 10.3348/jksr.2014.71.4.173.

14. Robinson K, Rajebi MR, Zimmerman N, Zeinati C. Postpancreaticoduodenectomy hemorrhage of unusual origin: Treatment with endovascular embolization and the value of preoperative CT angiography. J Radiol Case Rep. 2013;7(4):29-36. doi: 10.3941/jrcr.v7i4.1254. [PubMed: 23705050]. [PubMed Central: PMC3661432].

15. Weitz J, Koch M, Kleeff J, Muller MW, Schmidt J, Friess H, et al. [KauschWhipple pancreaticoduodenectomy. Technique and results]. Chirurg. 2004;75(11):1113-9. German. doi: 10.1007/s00104-004-0951-4. [PubMed 15517115].

16. Chong M, Freeny PC, Schmiedl UP. Pancreatic arterial anatomy: Depiction with dual-phase helical CT. Radiology.1998;208(2):537-42. doi 10.1148/radiology.208.2.9680589. [PubMed: 9680589].

17. Okada T, Yamaguchi M, Takahashi T, Izaki K, Uotani K, Sakamoto N, et al. Is embolization of the pancreas safe? Pancreatic histological changes after selective transcatheter arterial embolization with N-butyl cyanoacrylate in a swine model. Cardiovasc Intervent Radiol. 2012;35(1):161-7. doi: 10.1007/s00270-011-0130-1. [PubMed: 21547524]. 